\title{
Aspetti clinici ed epidemiologici dell'epatite $C$ nei pazienti in emodialisi
}

\author{
B. Di Iorio, G. Gaudiano, C. Altieri, V. Terracciano \\ Servizio di Emodialisi della USL $n^{\circ} 4$ del Lagonegrese, \\ Ospedali di Lauria e Maratea
}

identificazone di due virus associati all'epatite non A non B (nA-nB) (il virus dell'epatite E, epatite a trasmissione enterica o feco-orale molto diffusa nei Paesi in via di sviluppo, e il virus dell'epatite $C$, ritenuto il maggiore responsabile dell'epatite $\mathrm{nA}-\mathrm{nB}$ post-trasfusionale nonchè delle forme definite "community acquired") ha dato impulso alle ricerche per l'allestimento di metodi sierologici diagnostici.

Con la clonazione del genoma dell'HCV e il successivo allestimento di test immunodiagnostici mediante impiego di proteine virali ricombinanti è stato possibile, in breve tempo, disporre di una vasta messe di dati sulla biologia del virus, definendo meglio la storia naturale della malattia e tracciando il profilo epidemiologico dell'infezione (1-2).

\section{Caratteristiche del virus}

Il genoma virale è un RNA a filamento singolo composto da circa 9400 nucleotidi a polarità positiva e provvisto di envelope lipidico (Tab. I) $(1,2)$.

E organizzato in un solo gene preceduto in corrispondenza della porzione $5^{\prime}$ da una sequela di 320-340 nucleotidi che non è tradotta in proteina e che precede la regione codificante. Tale sequela di 320-340 nucleotidi probabilmente con- tiene le informazioni necessarie per la replicazione virale e per la trasmissione della poliproteina codificata di 30103011 aminoacidi che viene successivamente processata da proteasi virali e/o cellulari per dare origine alle diverse proteine del virus (Fig. 1) $(1,2)$.

L'RNA genomico, che può funzionare direttamente da RNA messaggero, codifica sia proteine strutturali, in corrispondenza della porzione 5 ' terminale, che non strutturali, in corrispondenza della regione intermedia e 3 ' terminale $(1,2)$.

Sulla base delle conoscenze riguardanti l'organizzazione molecolare del genoma, l'analisi delle sequenze nucleotidiche e le caratteristiche chimico-fisiche, il virus HCV viene oggi classificato nella famiglia delle FLAVIVIRIDAE, accanto ai Flavivirus e ai Pestivirus.

\begin{abstract}
Numerose segnalazioni indicano che $\mathrm{HCV}$ è geneticamente eterogeneo, essendo rappresentato da più sierotipi classificabili almeno in quattro genotipi maggiori (3). Ma altri genotipi sono in fase di caratterizzazione. Ciascun genotipo a sua volta comprende un numero variabile di sottotipi: questi hanno in comune oltre il $90 \%$ delle sequenze nucleotidiche del genoma e pertanto differiscono per meno del $10 \%$.

Inoltre le regioni $\mathrm{C}, \mathrm{NS} 3, \mathrm{NS} 4, \mathrm{NS} 5$ del genoma virale sono conservate in tutti i genotipi, per cui le proteine da esse codificate sono utilizzabili come antigeni per i test diagnostici (Fig. 1).

Esiste una differenza geografica nella distribuzione dei diversi genotipi. Il tipo I è più diffuso in USA ed Europa, i tipi II e III in Giappone e Cina, il tipo IV è se-
\end{abstract}

\section{TABELLA I - CARATTERISTICHE DEL VIRUS DELL'EPATITE C}

- Presenza di mantello lipidico

- Dimensione: 50-60 nm di diametro

- Capacità di indurre strutture citoplasmatiche tubulari in epatociti di scimpanzè sperimentalmente infettati

- Acido nucleico: RNA singola elica, polarità positiva, dimensioni di circa 9400 basi

- Tassonomia: Flavirus 


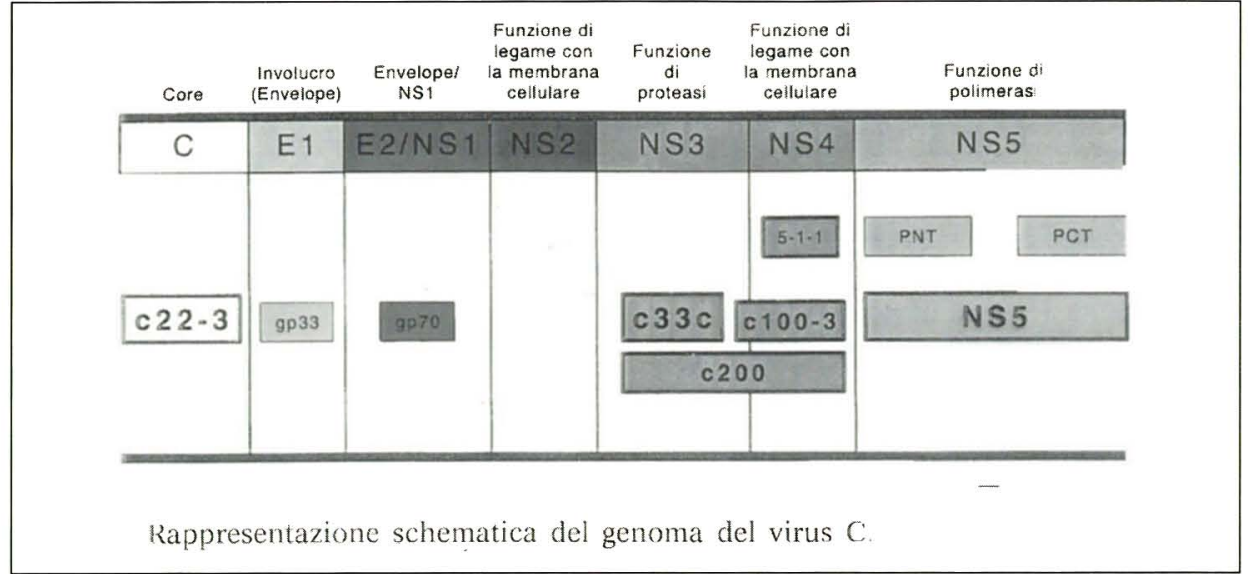

Fig. 1 - Rappresentazione schematica del genoma del virus $C$.

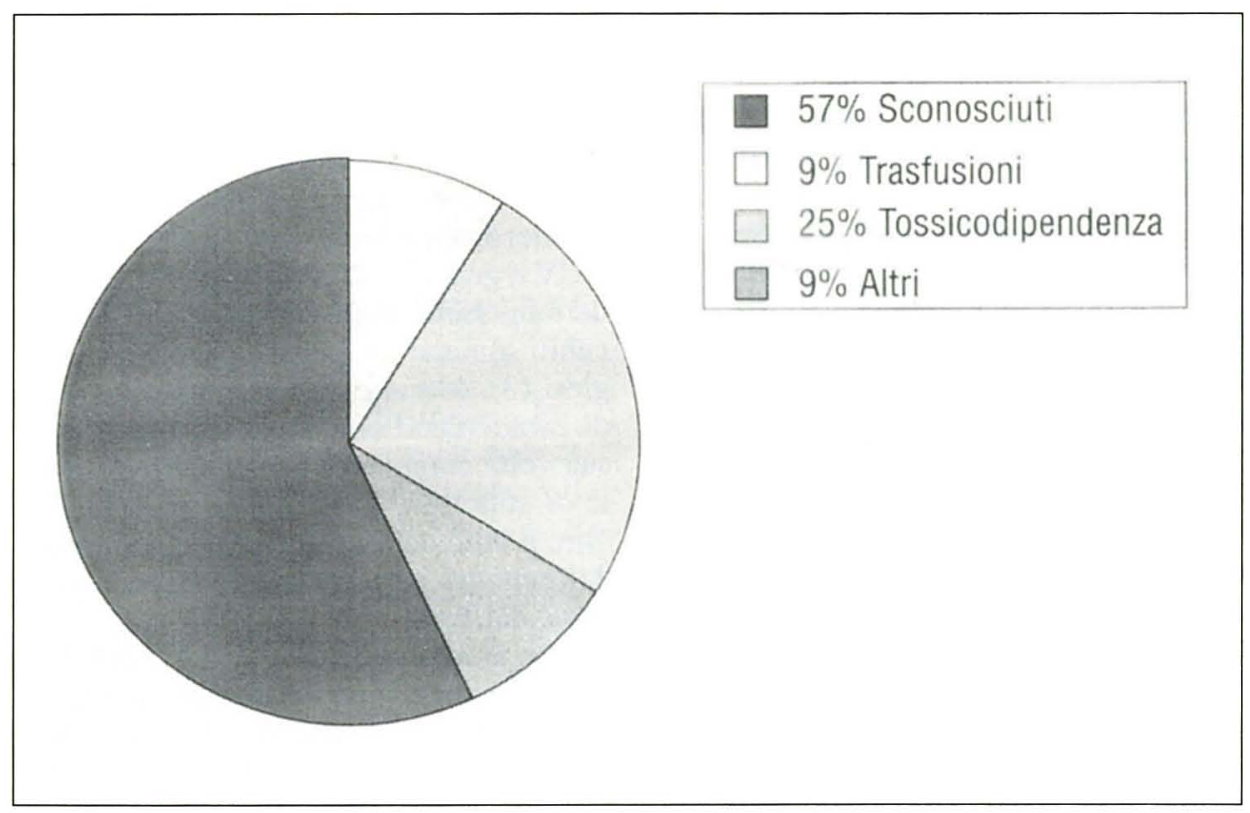

Fig. 2 - Fattori di rischio per epatite $C$ in Italia.

gnalato in Giappone, Egitto, Yemen. Secondo alcuni esperti il genotipo II corrisponde al ceppo selvatico di HCV da cui sarebbero derivati gli altri.

È dimostrato che in una singola area geografica possono circolare contemporaneamente diversi genotipi. In Europa, per es., sono presenti i genotipi I, II e III. In Italia ed Ungheria è stato segnalato anche il genotipo IV.

Studi di Piazza (4) hanno dimostrato che in Campania circolano tutti i 4 genotipi: nel $50 \%$ il genotipo I, nel $28.5 \%$ il III, nell' $8.5 \%$ il IV, nel $7.1 \%$ il I.

In alcuni soggetti sono presenti due o più genotipi diversi. Le varianti di HCV pos- sono causare reinfezione in soggetti già precedentmeente affetti da epatite $\mathrm{C}$.

TABELLA II - CARATTERISTICHE DELLE EPATITI VIRALI A CONFRONTO

\begin{tabular}{lccc}
\hline & HAV & HBV & HCV \\
\hline Sintomi gastroenterici & ++ & + & +- \\
Febbre & ++ & + & no \\
Ittero & ++ & + & +- \\
Poliatralgie & no & ++ & +- \\
Picco ALT & $>1000$ & $>1000$ & $<1000$ \\
Profilo ALT & monofasico & monofasico & polifasico \\
$\%$ Cronicizzazione & 0 & $2-5$ & $40-75$ \\
\hline
\end{tabular}
Figura 2 mostra i fattori di rischio per

\section{Aspetti clinici}

L'epatite acuta di tipo $C$ è una malattia paucisintomatica, raramente itterigena, con valori delle transaminasi che non superano nel picco di acuzie le 1000 U.I.

Il periodo di incubazione varia da 5 a 12 settimane. Inoltre l'andamento dell'epatite $\mathrm{C}$ è spesso caratterizzata da polifasicità del profilo delle transaminasi sieriche con più picchi di citolisi nel tempo (Tab. II).

Nei pazienti guariti si è osservato un calo progressivo sino a completa scomparsa degli anticopri anti C 100 entro 5 anni dalla fase acuta e scomparsa di tutte le reattività anticorpali nel $60 \%$ dei casi fra il $5^{\circ}$ e il $9^{\circ}$ anno.

Invece i pazienti con evoluzione cronica dimostrano nel tempo persistente positività per anti-HCV. La ricerca di $\mathrm{HCV}$ RNA nel siero mediante reazione polimerasica a catena (PCR) si dimostra il parametro più valido per la valutazione prognostica. La positività per HCV-RNA oltre il $3^{\circ}$ mese dall'esordio clinico della malattia risulta predittivo di cronicizzazione. Il decorso dell'epatite $\mathrm{C}$ cronica è sfavorevole con evoluzione in cirrosi e carcinoma con fasi di remissione transitoria e con il decorso della malattia tipicamente caratterizzato da un alternarsi di periodi di attività e di quiescenza bioumorale (5-7).

Un ulteriore aspetto clinico che merita un approfondimento riguarda il significato del rilievo positivo per anti-HCV in soggetti asintomatici con valori normali delle transaminasi sieriche. Va segnalata che è stata documentata (8) un'elevata prevalenza di epatite cronica in soggetti portatori asintomatici di anti-HCV indipendentemente dalle transaminasi.

Il virus $\mathrm{C}$ a diffusione ubiquitaria. $\mathrm{La}$ 
TABELLA III - PREVALENZA DELL'INFEZIONE DA VIRUS C NELLE CATEGORIE A RISCHIO

$\begin{array}{ll}>70 \% & \text { Emofilici } \\ & \text { Tossicodipendenti } \\ & \text { Epatiti croniche HBsAg-negative } \\ & \text { Epatiti post-trasfusionali } \\ & \text { Epatiti autoimmuni } \\ & \text { Alcolismo } \\ \text { Emodializzati } \\ \text { Epatiti croniche HBsAg-positive } \\ & \text { Contatti sessuali } \\ & \text { Trasmissione materno-fetale } \\ & \text { Diffusione familiare }\end{array}$

epatite $\mathrm{C}$ in Italia.

La presenza di HCV-RNA è stata dimostrata nella saliva dei pazienti con epatite C. Ciò indicherebbe che la saliva di questi pazienti può trasmettere l'infezione. Inoltre HCV-RNA è stato riscontrato nel liquido seminale, ascitico e nelle urine. Nelle urine in particolare la concentrazione di HCV-RNA è più bassa che nel sangue, ma occorre ugualmente cautela nel maneggiare i campioni di urine dei pazienti con epatite $\mathrm{C}$.

Le modalità di trasmissione di HCV sono uguali a quelle del virus dell'epatite B. La trasmissione per via parenterale e parenterale inapparente è stata accertata. La trasmissione orofecale non avviene.

È importante sottolineare che anti-HCV sono stati evidenziati nel $70 \%$ di soggetti con ca. epatocellulare, nel $30 \%$ di soggetti con Epatite Cronica Attiva (ECA) o cirrosi di origine alcolica, e nel $60 \%$ di ECA autoimmuni.

Recentemente è stata segnalata l'associazione tra $\mathrm{HCV}$ e patologie extraepatiche quali la S. di Sjogren e la Crioglobulinemia Mista Essenziale. Vi sono evidenze scientifiche che in quest'ultima malattia HCV possa svolgere un ruolo etiopatogenetico. HCV si trasmette anche per via sessuale. Gli studi apparsi in letteratura indicano che tale rischio presenta ampie oscillazioni nella popolazione.

La prevalenza di HCV nei donatori di sangue è abbastanza omogenea nelle diverse nazioni $(0.5-2.3 \%)$ ad eccezione dell'Egitto dove risulta più alta $(10.9 \%)$. Inoltre anche per quanto riguarda il virus $\mathrm{C}$, alcune categorie presentano una prevalenza estremamente alta (Tab. III): essa supera il $70 \%$ negli emofilici, nei tossicodipendenti, nei soggetti con epatite cronica HBV-negativa o con epatite post trasfusionale; è del 30-60\% nei pazienti in emodialisi, negli alcolisti, nei pazienti affetti da epatite autoimmune o da epatite HBV-positiva.

\section{Diagnosi}

La diagnosi sierologica dell'infezione da HCV si basa principalmente sulla presenza o meno nel siero del paziente di anticorpi specifici diretti contro gli antigeni strutturali del core e antigeni non strutturali dell'HCV (4).

Il test diagnostico più comune è quello immunoenzimatico di II generazione (ELISA II). Tale test evidenzia C 22-3 (core) e C 100-3 e C33-c (antigeni non strutturali).

La conferma avviene mediante test di recombinant immunoblotting assay (RIBA II) che evidenzia $C 22-3$ e gli antigeni non strutturali C 100-3, C 33-c, 5-11.
La determinazione di HCV-RNA mediante PCR è una metodica altamente sensibile in grado di rilevare anche bassissime concentrazioni di acido nucleico del virus $\mathrm{C}$. La metodica non è di facile esecuzione e non tutti i laboratori sono in grado di eseguirla correttamente.

Recentemente è stato introdotto il test ELISA III generazione ed è anche disponibile in commercio il RIBA III.

\section{Cenni terapeutici}

Come per l'epatite B le sostanze usate con maggior successo nella terapia dell'epatite C sono state gli alfa interferoni (IFN-alfa). Si ottiene una remissione clinica, laboratoristica e istologica nel 50$70 \%$ dei pazienti trattati con 3-6 milioni di $U$ per tre volte la settimana per 6 mesi (9-11). L'interferone ha attività antivirale, antiproliferativa e immunomodulante. La probabilità di evoluzione cronica può venire ridotta dal 79 al $9 \%$.

Sono possibili delle ricadute alla sospensione del trattamento. La remissione duratura della malattia e scomparsa della malattia si verifica in circa il $20 \%$ dei pazienti trattati (Fig. 3).

Terapie a più lungo termine (12 mesi) mostrano una maggiore efficacia (12), così come la contemporanea somministrazione di $\mathrm{N}$-Acetilcisteina riduce la percentuale di non responder alla terapia con interferone potendosi anche ridurre la dose a 1 milione di U. (13).

È sempre più attiva la ricerca di nuovi farmaci o associazioni terapeutiche che possano migliorare i risultati finora ottenuti dalla farmacoterapia.

IFN-beta presenta una maggiore tollera-

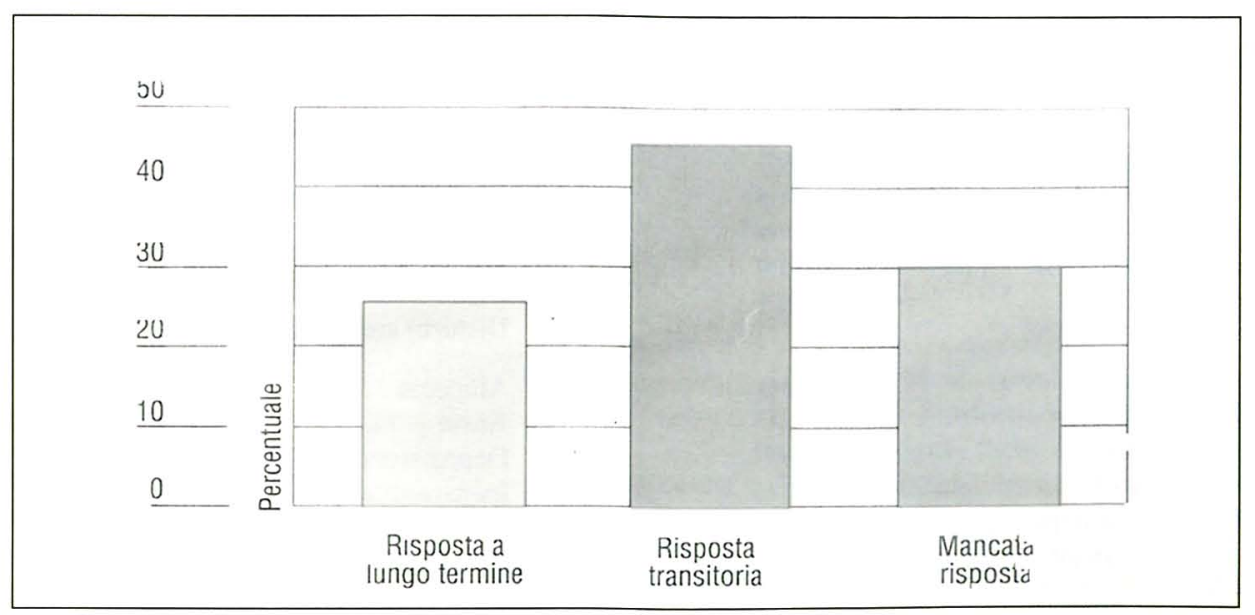

Fig. 3 - Risposta alla terapia con $\alpha$ interferone di pazienti anti-HCV positivi. 
bilità rispetto a IFN-alfa e una minore incidenza di effetti collaterali (14-15). IFN-beta è usato in caso di intolleranza a IFN-alfa al dosaggio di 3-6 milioni di unità a gg. alterni per 6-12 mesi (Fig. 4). Recentemente (16) è stato effettuato uno studio sull'efficacia e la tollerabilità del trattamento di IFN-beta e inosina-pranobex. Quest'ultimo è un farmaco antivirale che oltre ad essere attivo contro i virus a DNA e RNA possiede effetti immunomodulanti agendo da induttore di interleukina-2 e del suo recettore. Un dato interessante dello studio è la dimostrazione di minore incidenza di ICAM-1 (molecola di adesione intercellulare) nei soggetti responders rispetto a quelli non responders. La combinazione IFN-beta e inosina-pranobex sembra più efficace rispetto al solo trattamento con IFN. Inoltre poiché è stato osservato che l'espressione di ICAM-1 mediata dalle citochine è ridotto dall'uso dei corticosteroidi, è possibile ipotizzare che la loro associazione alla terapia con IFN e antivirali possa garantire un migliore effetto terapeutico (16). Tutti i pazienti hanno manifestato in modo transitorio modesti sintomi simil-influenzali, facilmente dominati da dosi convenzionali di paracetamolo. Come effetti collaterali si possono avere segni di modesta e reversibile mielosoppressione. Inconvenienti maggiori possono essere ipertiroidismo, depressione psichica e infezioni. Sono stati segnalati inconvenienti di minore importanza come riduzione del peso corporeo, caduta dei capelli, modesta apatia, anoressia (Tab. IV).

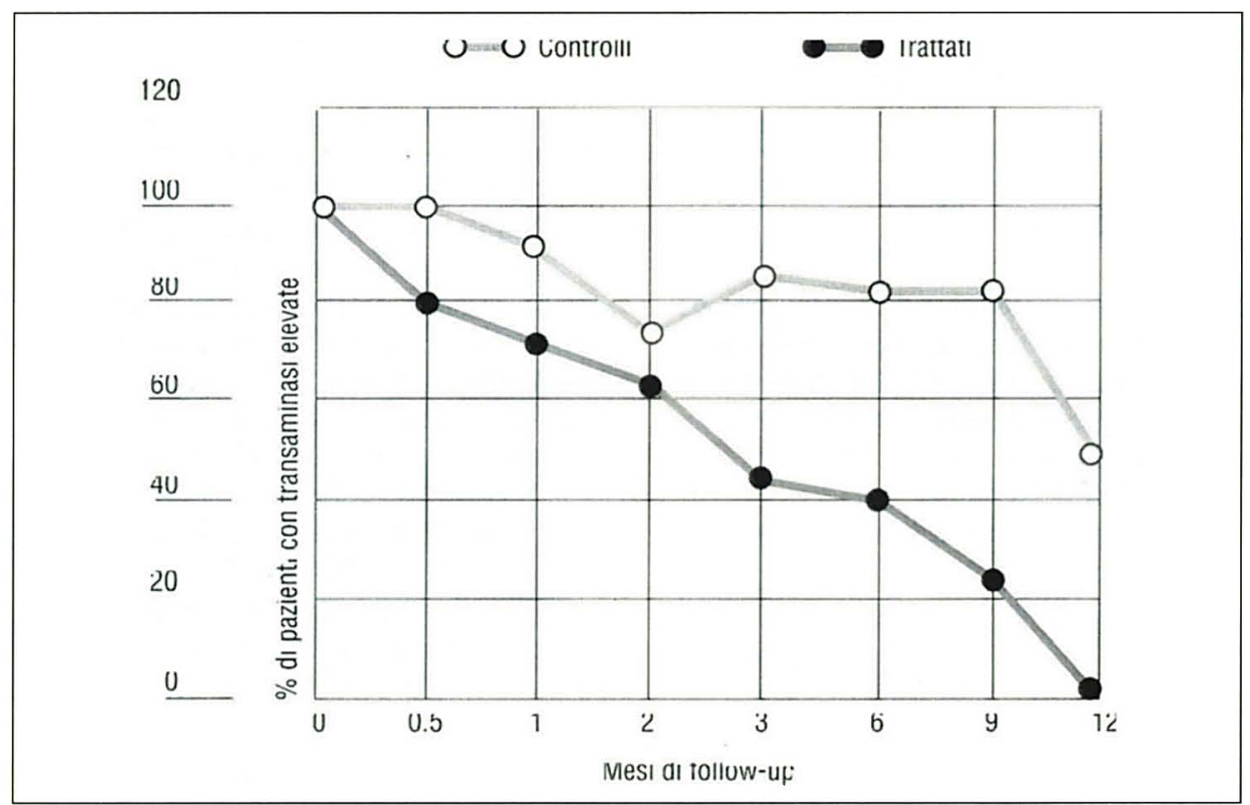

Fig. 4 - Percentuale di pazienti con ipertransaminasemia (ALT) nel gruppo di trattamento con $\beta$ interferone e di controllo. TABELLA IV - EFFETTI COLLATERALI (\%) DELLA TERAPIA CON INTERFE-
RONE (IFN)

COSTANTI

$100 \%$

COMUNI

$30-60 \%$
FREQUENTI 10-20\% Alopecia

FREQUENTI 10-20\% Alopecia

Sindrome simil-influenzale

Mielodepressione

Perdita di peso

Disturbi del sonno

Disturbi concentrazione

Depressione

Ipertiroidismo

Anticorpi anti-IFN

RARI

$1-5 \%$
Disfunzioni sessuali

Reazioni autoimmuni

\section{Cenni epidemiologici in emodialisi}

Gli emodializzati sono un gruppo di pazienti ad alto rischio per la possibilità di contrarre infezioni virali nosocomiali a trasmissione parenterale e quindi epatite C (17-20). In letteratura la prevalenza dei soggetti HCV positivi varia in un range assai vasto: dall' $1 \%$ al $40 \%$ con ampie oscillazioni geografiche (21-24). In particolare la percentuale varia dall' $1.7 \%$ del Lussemburgo e l' $1.9 \%$ dell'Irlanda al $35.6 \%$ del Marocco e al 49.5\% della Polonia (25).

In Italia la percentuale di positività per anti-HCv nei pazienti in emodialisi è del $32.2 \%$ (25). Anche qui vi è un ampio range di oscillazione: dal $12 \%$ di Rovigo (17), al 15.5 di Palermo (26), al 24\% di Prato e Pistoia (27), al 30.5, 35.8, 36.7 di Adria, Chioggia, Mestre (18) al 38\% di Casale Monferrato (28).

\section{La nostra esperienza}

Abbiamo testato con test screening di II generazione (EIA-Abbott) e valutato le reattività sieriche contro diversi antigeni ricombinanti (Matrix HCV-Abbott) 89 pazienti (53 M, $36 \mathrm{~F})$ (26).

22/89 pazienti sono risultati HCV positivi ( $24.72 \%$ della popolazione).

L'età anagrafica dei pazienti HCV positivi era $56.37 \pm 14.27$ anni mentre nei pazienti HCV negativi era 58.21 \pm 15.62 (NS); l'età dialitica era 101.88 \pm 68.16 mesi vs $48.51 \pm 48.03$ ( $\mathrm{p}<0.001)$.

Il $66.70 \%$ dei pazienti HCV positivi aveva ricevuto almeno 1 emotrasfusione, mentre nei pazienti HCV negativi solo il $36.36 \%$ di essi era stato trasfuso $(\mathrm{p}<0.05)$.

I pazienti $\mathrm{HCV}$ positivi presentavano transaminasi costantemente elevate nel $10.21 \%$ dei casi, occasionalmente nel $24.57 \%$, sempre normali nel $65.22 \%$. Per transaminasi elevate si intende un valore almeno doppio del limite di riferimento di normalità.

La Tabella V mostra il comportamento dei pazienti HCV positivi nei confronti dei vari antigeni testati: il $100 \%$ di essi presenta positività per Core, il $90.90 \%$ per NS-3, il 63.64\% per NS-4 YEAST, il $68.18 \%$ per NS-4 COLI.

I dati del nostro studio confermano che $\mathrm{i}$ fattori di rischio più importanti sono 
TABELLA V - FREQUENZA DEGLI ANTIGENI NEI PAZIENTI HCV POSITIVI DI LAURIA (29)

\begin{tabular}{lcccc}
\hline Antigeni & CORE & NS-3 & NS-4 YEAST & NS-4 COLI \\
Pazienti $^{\circ}$ & 22 & 20 & 14 & 15 \\
$\%$ & $(100)$ & $(90.90)$ & $(63.64)$ & $(68.18)$ \\
\hline
\end{tabular}

l'età dialitica $(\mathrm{p}<0.001)$ e la frequenza di trasfusioni $(\mathrm{p}<0.05)$.

La frequenza di emotrasfusioni nei pazienti $\mathrm{HCV}$ positivi è stata di 1.6 trasfusione/paziente, mentre nei pazienti HCV negativi solo di 0.33 . Inoltre la frequenza e la distribuzione delle trasfusioni per età dialitica mostra un'omogenea distribuzione dei pazienti. L'uso dell'eritropietina ha notevolmente ridotta la frequenza delle emotrasfusioni, ma rimane la possibilità di contaminazioni inapparenti dei pazienti attraverso l'uso improprio di materiale disposable e dei monitors: 4 pazienti HCV positivi non hanno mai ricevuto trasfusioni nè hanno rivelato contatto anamnestico con fonti di contaminazioni extra-dialisi. In un paziente è probabile come fonte di contaminazione la manovra per impianto di pace-maker e in un secondo la possibilità di aver contratto il virus dopo un ciclo di dialisi-vacanze extra-regione in ambiente privato. In conclusione è da sottolineare, anche nella nostra popolazione, l'elevata percentuale $(65.22 \%)$ di pazienti HCV positivi che non hanno mai presentato (nel periodo di osservazione, che per alcuni di essi è superiore ai 60 mesi) segni di citolisi epatica. Va sottolineato che è stata documentata (8) un'elevata prevalenza di epatite cronica in soggetti portatori asintomatici di anti-HCV indipendentemente dall'elevazione delle transaminasi.

\section{Conclusioni}

$\mathrm{Al}$ di là di considerazioni terapeutiche circa l'uso farmacologico di interferone nei pazienti dializzati, esiste un problema di ordine infettivologico: la necessità di isolamento di questi pazienti allo stesso modo dei portatori di virus $\mathrm{B}$ o meno (30).

Crediamo che l'elevato numero di pazienti interessati e le varie tecnologie impiegate nel trattamento limitino enorme- mente le possibilità pratiche di isolamento se non in pochi centri attrezzati.

Ancora bisogna considerare i limiti attuali del test per la determinazione di anti-HCV; l'intervallo tra esposizione al virus e comparsa degli anticorpi può essere assai lungo; la presenza di antiHCV non significa necessariamente una condizione di viremia (31).

Il nostro approccio al problema è quello di riservare sempre gli stessi monitors ai pazienti HCV positivi senza che tali pazienti siano isolati dal resto della popolazione dialitica. Importante è la sterilizzazione chimica dopo ogni trattamento emodialitico (32-35).

È chiaro che il problema diventa scottante in quei centri di dialisi (sempre più numerosi) dove l'enorme affluso di pazienti implica l'istituzione di tre turni di dialisi giornalieri, rendendo ovviamente ridotti i tempi interdialitici.

\section{BIBLIOGRAFIA}

1 .

Choo QL, Kuo G, Weiner L, et al. Isolation of a cDNA clone derived from a blood-borne non-A, non$B$ viral hepatitis genome. Science 1989; 244: 359-62.

2. Kuo G, Choo QL, Weiner L, et al. An assay for circulating antibodies to a major etiologic virus of human non-A, non-B viral hepatitis genome. Science 1989; 244: 362-4.

3. Houghton M, Weiner A, Han $\mathrm{J}$, et al. Molecular biology of the hepatitis $\mathrm{C}$ viruses. Implication for diagnosis, development and control of viral disease. Hepatology 1991; 14: 381-8.

4. Piazza M. Epatite virale acuta e cronica. Ghedini ed. Milano, 1994.

5. Marchesi D, Anci C, Poletti E, et al. Outbreak of non-A, non-B hepatitis in centre hemodialysis patients: a retrospective analysis. Nephrol Dial Transpl 1988; 3: 795-9.

6. Alter H, Purcell R, Shih J, et al. Detection of antibody to hepatitis $\mathrm{C}$ virus in prospectively followed trasfusion recipients with acute and chronic non-A, non-B hepatitis. New Engl J Med 1989; 321: 1494500 .

7. Di Iorio B, Giaudiano G, Altieri $C$, et al. Epatite $\mathrm{C}$ nei pazienti in dialisi. Aspetti clinici ed epidemiologici. Medico e Paziente 1994; 4: 19-21.

8. Esteban JI, Gonzales A, Hernandez JM, et al. Evaluation of antibodies to hepatitis $\mathrm{C}$ virus in a study of trasnfusion associated hepatitis. New Engl J Med 1990; 323: 1107-12.

9. Kagawa T, Morizane T, Saito $\mathrm{H}$, et al. A randomized, controlled trial of weekly administration of lymphoblastoid interferon in patients with chronic hepatitis $\mathbf{C}$. J of Hepatology 1993; 17: 91-6.

10. Milella M, Antonelli G, 
Santantonio T, et al. Neutralizing antibodies to recombinant alpha-interferon and response to therapy in chronic hepatitis $\mathrm{C}$ virus infection. Liver 1993; 13: 146-50.

11. Castilla A, Camps-Bansell J, Civeira MP, Prieto J. Lymphoblastoid alfa-interferon for chronic hepatitis $\mathrm{C}$ : a randomized controlled study. Am J Gastroenterology 1993; 2: 233-9.

12. De Alava E, Camps J, PardoMindàn $\mathbf{J}$, et al. Histological toucome of chronic hepatitis $\mathrm{C}$ treated with a 12-month course of lynphoblastoid alfa interferon. Liver 1993; 13: 73-9.

13. Beloqui $\mathrm{O}$, Prieto J, Suàrez $\mathrm{M}$, et al. N-acetyl Cysteine enhances the response to alfa-interferon in chronic hepatitis C: a pilot study. $\mathbf{J}$ of Interferon Research 1993; 13: 279-82.

14. Omata M, Yokosuka O, Takano S, et al. Resolution of acute hepatitis $\mathrm{C}$ after therapy with natural beta interferon. Lancet 1991; 338: 914-5.

15. Calleri G. Beta interferone nell'epatite acuta di tipo C. Mediterranean Journal of infection and parasitic diseases 1994; (S) IX: 28-33.

16. Sansonno D, Dammacco F. Terapia di associazione con interferone beta e inosina-pranobex dell'epatite cronica da HCV. In Epatite Virale: prevenzione e terapia. Italia/ Egyptian Symposium. Marrakech 15-16 dic. 1992, 79-83.

17. Salvadori M, Ippolito E, Aterini S, et al. Epatite C. Indagine epidemiologica in 2 centri di dialisi e proposte di profilassi. Gior Ital Nefrol 1991; 8: 347-51.

18. Gessoni G, Manoni F, Nordio B, et al. Studio policentrico sulla sierologia dell'HCV in emodializzati veneti: evidenze di modalità di infezione diverse dalla via trasfusionale. Gior Ital Nefrol 1992; 9: 203-8.

19. Jeffers L, Perez G, De Medina $M$, et al. Hepatitis C infection in two urban hemodialysis units. Kidney Int 1990; 38: 320-2.

20. Dentico P, Buongiorno R, Volpe A, et al. Prevalence and incidence of hepatitis $\mathrm{C}$ virus in hemodialysis patients: study of risk factors. Clin Nephrology 1992; 38: 4952.

21. Esteban J, Esteban R, Viladomiu L, et al. Hepatitis $\mathrm{C}$ virus antibodies among risk group in Spain. Lancet 1989; i: 294-7.

22. Gilli P, Moretti M, Soffritti S, Merini C. Anti HCV positive patients in dialysis units. Lancet 1990; ii: $243-4$.

23. Hruby Z, Sliwinski I, Molin $M$, et al. High prevalence of antibodies to hepatitis $\mathrm{C}$ virus in three haemodialysis centres in south-western Poland. Nephr Dial Transpl 1993; 8: 740-3.

24. Roggerdorf M, Deinhard F, Rasshofer R, et al. Antibodies to hepatitis C virus. Lancet 1989; ii: 324-5.

25. Raine AEG, Margreiter R, Brunner FS, et al. Report on management of renal failure in Europe, 1991. Nephr Dial Transpl 1992; 7 (S2): 7-35.

26. Mancino C, Caputo F, Sparano $\mathrm{V}$, et al. Prevalenza dell'infezione da HCV in un centro di emodialisi. Nefrol Dial Trapianto, Wichtig ed., Milano, 1990; 1: 333-6.

27. Fabrizi F, Raffaele L, Bacchini $\mathrm{G}$, et al. Antibodies to $\mathrm{HCV}$ and transaminase concentration in chronic haemodialysis patients; a study with second-generation assays. Nephr Dial Transpl 1993; 8: 744-7.

28. Vaggelli G, Guaschino R, Calabrese G, et al. Anticorpi anti $\mathrm{HCV}$ in unità dialitica ad alta incidenza di epatite non-A, non-B. Nefrol Dial Trapianto, Wichtig ed. Milano, 1990; 1: 71-3.

29. Di Iorio B, Mitidieri-Costanza $\mathrm{O}$, Gaudiano G, et al. Anti-HCV in una popolazione dialitica lucana. Studio con test di II generazione e analisi dei fattori di rischio. G I N 1994; 11: 219-21.

30. Gilli P, Bergani M, Soffritti S. Considerazioni sulla prevalenza di pazienti anti HCV positivi nei centri di dialisi. Gior Ital Nefrol 1990; 7: 271-2.

31. Alter MJ, Sampliner RE. Hepatitis C and miles to go before we sleep. New Engl J Med 1989; 321: 1538-41.

32. Gilli P, Soffritti S, De Paoli E, et al. Prevenzione dell'epatite $C$ nei Centri Dialisi. Abstract XXXV Congresso Società Italiana di Nefrologia, Bari 18-21 maggio 1994 , p. 70.

33. Lombardi M, Cerrai T, Dattolo $P$, et al. È la membrana dialitica una barriera sicura contro il virus C? Abstract XXXV Congresso Società Italiana di Nefrologia, Bari 18-21 maggio 1994, p. 71.

34. Papalia T, Cassani S, Martire $\mathrm{R}$, et al. Misure preventive dell'infezione $\mathrm{HCV}$ in un centro dialisi ad assistenza limitata. Abstract XXXV Congresso Società Italiana di Nefrologia, Bari 18-21 maggio 1994, p. 75.

35. Fabrizi F, Di Filippo S, Guarnori I, Locatelli F. Incidenza di sieroconversione da anti-HCV in pazienti emodializzati cronici: studio prospettico. Abstract XXX.V Congresso Società Italiana di Nefrologia, Bari 18-21 maggio 1994; p. 68. 